\title{
Utilisation de la photogrammétrie terrestre et de la télémétrie sans réflecteur pour la mesure de l'érosion de petits bassins-versants et comparaison avec les mesures de transport solide à l'émissaire
}

\author{
Y. Egels $\left({ }^{1}\right)$, M. Kasser $\left({ }^{1}\right)$, M. Meunier $\left({ }^{2}\right)$, T. Muxart $\left({ }^{3}\right)$ et C. Guet $\left({ }^{2}\right)$ \\ PIREN Milieu rural. Programme «érosion»
}

\begin{abstract}
La perte en terre due à l'érosion s'exerçant sur une unité territoriale peut se faire en mesurant le transport solide dans un chenal (émissaires, "gouttières" de parcelles) ou l'ablation et l'accumulation superficielles le long de piquets-repères. Dans le premier cas, on obtient des valeurs globales intégrant l'ensemble des phénomènes s'étant produits dans l'espace entre 2 dates. Dans le second cas, on apprécie l'ampleur et le sens des transformations locales du milieu, selon la grille d'implantation des piquets, d'où l'on tire la tranche moyenne de matériau érodé (ou déposé) dans le secteur, en un temps donné. Toutefois, la présence même des piquets risque de fausser les mesures.

Pour s'affranchir de cet inconvénient on a utilisé des méthodes topographiques (photogrammétrie terrestre et télémétrie sans réflecteur) qui saisissent, à la fois, les variations locales et globales du milieu physique, sans le perturber. Une opération-test multiorganismes (CNRSUA 141, IGN, CEMAGREF, PIREN) a été menée sur le site des bassins-versants expérimentaux de Draix du CEMAGREF. Il s'agissait de contrôler la validité d'emploi de ces méthodes pour la métrologie de l'érosion, notamment, en évaluant le volume moyen annuel érodé et en le comparant avec celui mesuré dans
\end{abstract}

(1) IGN, 2, av, Pasteur, 94160 St-Mandé.

(2) CEMAGREF, Domaine Universitaire, BP 76, 38402 StMartin-d'Hères.

( $\left.{ }^{3}\right)$ CNRS, UA 141, 1, pl. A. Briand, 92190 Meudon. le collecteur de la ravine. Dans ce qui suit, seront décrits le site et le dispositif de mesures du transport solide, puis les deux méthodes topographiques testées et les résultats obtenus. Enfin, seront discutés l'intérêt de ces méthodes et le modèle physique implicite permettant de comparer ces résultats avec ceux des mesures du transport solide.

1. Le site des bassins-versants de Draix et la mesure des transports solides

\subsection{Description sommaire}

Les bassins-versants expérimentaux de Draix ont été décrits par ailleurs [1]. Situés à $15 \mathrm{~km}$ au N-E de Digne, entre 800 et $1300 \mathrm{~m}$, ils se développent dans des marnes et comportent 3 bassins de 100 ha, un de 8 ha, et une ravine élémentaire ( $\mathrm{La}$ Roubine) de $1300 \mathrm{~m}^{2}$, choisie pour l'expérimentation. Pluies, débits liquides et solides sont mesurés depuis plusieurs années. L'érosion est importante (60 à $\left.90 \mathrm{~m}^{3} / \mathrm{ha} / \mathrm{an}\right)$ et les pentes fortes $(70 \%)$, ce qui permet de saisir une partie significative du bassin versant à partir des appareils topographiques situés au sol.

\subsection{La mesure des transports solides sur la Roubine}

Les débits solides et liquides de la Roubine sont mesurés depuis 1983 [2]: une fosse de $4 \mathrm{~m}^{3}$ barrée par une grille

\footnotetext{
Use of terrestrial photogrammetry and reflectorless electronic telemetry (EDM) for erosion measurements in small catchments and comparison with measurements of bed and suspended loads of the brook
}

Terrestrial photogrammetry and reflectorless electronic telemetry were tested as measures of erosion in a first order catchment cut into marl bedrock at Draix (Southern Alps, France). These methods have been used to evaluate precisely $(\leqslant 1 \mathrm{~cm})$, by periodic survey, the amount of slope changes, without disturbing the environment. The mean annual volume of erosion has been estimated from these data and compared with measurements of bed and suspended loads of the stream. 
de $20 \mathrm{~mm}$ de maille stocke l'essentiel des transports solides; en aval un déversoir triangulaire assure la correspondance hauteur-débit ; le niveau est mesuré par deux limnigraphes (à flotteur et à ultrasons aériens) ; enfin un préleveur d'échantillons, asservi à un limnigraphe permet de connaitre la concentration moyenne de MES traversant la grille. Par cubage hebdomadaire, on obtient un volume de dépôts, correspondant, le plus souvent, à une seule pluie, et un hydrogramme solide (sauf en cas de défection des appareils).

\subsection{Les résultats et l'incertitude sur les mesures}

Entre les deux relevés par les méthodes topographiques, $(17 / 10 / 85$ et $4 / 11 / 86)$, le volume total des dépôts piégés a été de $13,939 \mathrm{~m}^{3}$. Les 3 premières mesures correspondent à des coulées boueuses dues au dégel, ou à une saturation longue entraînant une reptation et/ou une solifluxion ; le préleveur étant hors service, on a admis que le volume de MES était nul. Il n'en est pas de même pour les 9 autres relevés. Pour les 5 épisodes complètement suivis, le rapport (volume de MES)/(volume dans la fosse) varie entre 8 et $18 \%$ (moyenne $=10 \%$ ). En adoptant une formule corrective [3] pour les crues mal observées, on trouve $1,823 \mathrm{~m}^{3}$ de MES supplémentaires et un volume total de transport solide de $15,8 \mathrm{~m}^{3}$.

L'incertitude des mesures a deux causes : l'extrapolation du volume transité pour les crues mal observées et l'utilisation d'unités de mesures différentes pour les MES (poids) et les dépôts piégés (volume). Faute de connaittre la variabilité de la densité des dépôts, une valeur de 1,7 a été adoptée pour comparer les résultats.

\section{Les méthodes topométriques utilisées}

\subsection{La photogrammétrie}

Schématiquement, une photographie "métrique ", issue d'une caméra ne déformant pas l'image, représente une archive d'où l'on tire des mesures de directions entre le centre de l'optique et n'importe quel détail du cliché. L'exploitation stéréoscopique se résume géométriquement à l'intersection dans l'espace de deux « faisceaux perspectifs ": ensembles de droites passant par les centres optiques et les points homologues de deux clichés. Des données complémentaires (points côtés) permettent le calcul de la position des points sur les images. Ce procédé, généralement aéroporté, peut être mis en cuvre au sol, en cas de relief suffisant. Il permet une métrologie, sans contact, d'objets de tailles variées. En choisissant les paramètres techniques (nombre de points de vue, échelle des clichés, ...), on peut déterminer un nombre illimité de points avec une très bonne précision (quelques $\mathrm{mm}$ sur plusieurs centaines de $\mathrm{m}$ ou le $\mathrm{cm}$ sur plusieurs $\mathrm{km}$ ).

Pour la Roubine, les prises de vue ont duré une demijournée avec des conditions météorologiques restrictives (éclairage diffus, pas d'ombres portées). Elles ont été effectuées avec une caméra métrique WILD-P 31 et pour l'exploitation des clichés avec le restituteur analytique TRASTER 1 de MATRA (durée 1 semaine).

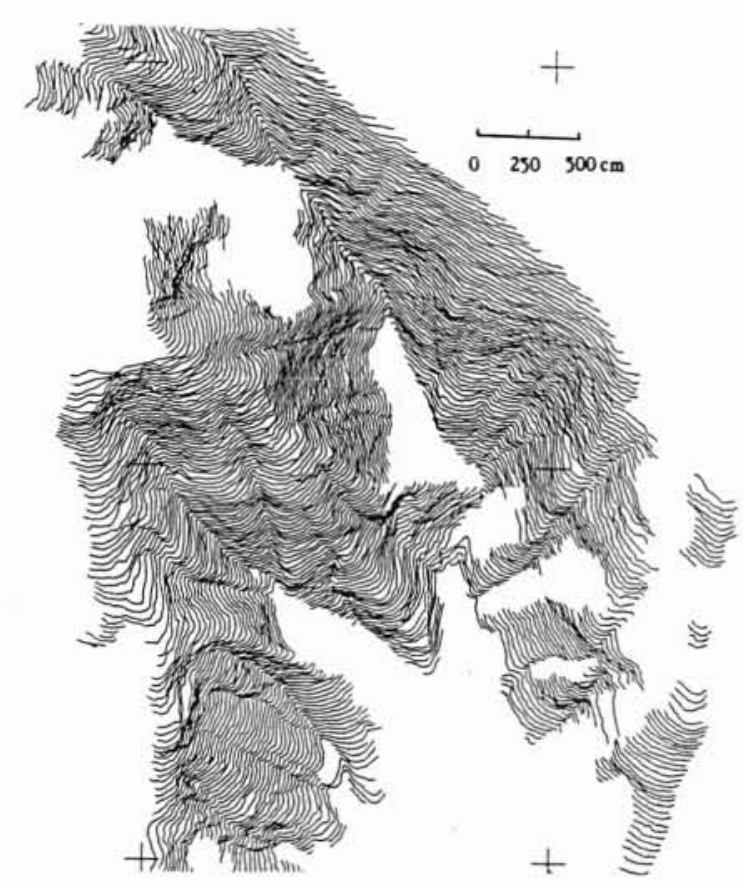

1. Extrait du plan de la Roubine en 1985, établi par photogrammétrie (échelle obtenue: $1 / 250^{\circ}$; équidistance des courbes : $20 \mathrm{~cm}$ ).

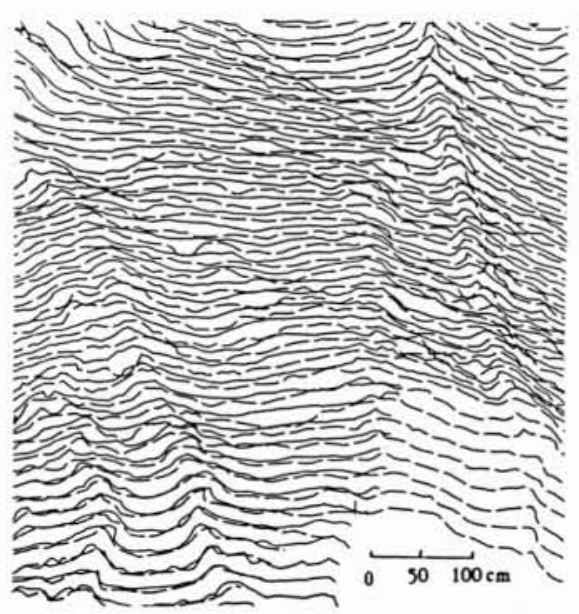

2. Exemple des courbes de niveau établies en 1985 - tireté - et 1986 -trait plein (échelle obtenue: $1 / 50^{\circ}$; équidistance des courbes : $20 \mathrm{~cm}$ ).

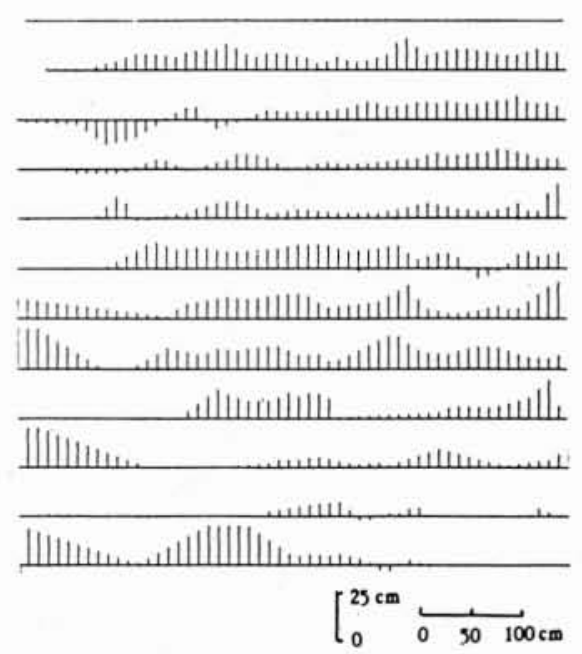

3. Exemple de répartition des écarts entre 1985 et 1986 (échelle planimétrique obtenue: $1 / 50^{\circ}$; échelle des écarts: $1 / 25^{e}$; équidistance des profils : $50 \mathrm{~cm}$ ). 


\section{Tableau 1}

$N=$ Nombre de mesures ; $x=$ moyenne de la distance calculée et $\sigma=$ écart-type.

\begin{tabular}{|c|c|c|c|c|c|c|c|c|c|}
\hline & & Dépôt & & & Ablatio & & & Total & \\
\hline & $\mathrm{N}$ & $\underset{\mathrm{m}}{\overline{\mathrm{x}}}$ & $\underset{\mathrm{mm}}{\sigma}$ & $\mathrm{N}$ & $\underset{\mathrm{mm}}{\overline{\mathbf{x}}}$ & $\underset{\mathrm{mm}}{\sigma}$ & $-\mathrm{N}$ & $\begin{array}{c}\overline{\mathrm{x}} \\
\mathrm{mm}\end{array}$ & $\underset{\mathrm{mm}}{\tau}$ \\
\hline $\begin{array}{c}\text { Station } \\
\text { basse }\end{array}$ & 31 & 24,9 & 40 & 299 & $-29,2$ & 17,9 & 330 & -24 & 26 \\
\hline $\begin{array}{l}\text { Station } \\
\text { haute }\end{array}$ & 24 & 25 & 27,6 & 69 & -28 & 23,0 & 93 & $-14,4$ & 33,4 \\
\hline
\end{tabular}

Tableau 2

\begin{tabular}{|c|c|c|c|c|}
\hline & \multicolumn{2}{|c|}{ Station basse } & \multicolumn{2}{|c|}{ Station havte } \\
\hline & érodé & seposé & érodé & déposé \\
\hline votume de terre $\left(m^{3}\right)$ & $-5,774$ & 0.328 & -2.627 & 0.598 \\
\hline Surface développée $\left(m^{2}\right)$ & 252.637 & 40.529 & 121.671 & 36.574 \\
\hline $\begin{array}{l}\text { Epaisseur correspondante } \\
\text { (m) }\end{array}$ & -22.8 & 8.0 & -21.6 & 10.6 \\
\hline foalsseur $(m)$ & \multicolumn{2}{|c|}{$-18,6$} & \multicolumn{2}{|c|}{-11.38} \\
\hline Aolation verticale $(\mathrm{mm})$ & \multicolumn{2}{|c|}{$-22,7$} & \multicolumn{2}{|c|}{$-13,9$} \\
\hline
\end{tabular}

\subsection{La télémétrie sans réflecteur}

Les distancemètres utilisent généralement un faisceau infrarouge modulé dont la mesure du déphasage sur la modulation entre l'aller et le retour permet de calculer la distance. Depuis peu, une autre technique consiste à analyser une série d'impulsions lumineuses brèves fournies par une diode laser. En mesurant le temps de vol mis par chaque impulsion pour faire l'aller-retour entre l'appareil et la cible, on détermine la distance avec une précision de quelques $\mathrm{mm}$. L'intérêt de la méthode est aussi la mesure sur cibles non coopératives, sans réflecteur (murs, sol, ...). Les appareils commercialisés par Fennel et par Wild permettent des mesures sans contact jusqu'à des portées importantes $(50 \mathrm{~m}, 100 \mathrm{~m}$, ou plus) sans être dangereux pour l'œil humain. Un télémètre FENNEL FET 2, monté sur un théodolite électronique, aimablement prêté par la Société le Pont Equipements a été utilisé. Trois repères ont été implantés : deux pour disposer le télémètre à une hauteur constante et le troisième pour orienter les mesures angulaires.

\section{Résultats des méthodes topographiques}

\subsection{La photogrammétrie}

Compte tenu des zones visibles sur les clichés de 1985 et de 1986 , seuls $500 \mathrm{~m}^{2}$ sur $1300 \mathrm{~m}^{2}$ ont fait l'objet de mesures comparatives, avec une prédominance des zones de pentes fortes en haut des versants. La précision est d'environ 1 à $2 \mathrm{~mm}$ en $x, y, z$, portant sur plusieurs milliers de points. Le résultat fourni est un levé au 1/50 avec une équidistance de $20 \mathrm{~cm}$; l'écart entre les positions temporelles successives d'une courbe de niveau traduit le déplacement de la surface entre les deux relevés (fig. 1 à 3). A partir des données numériques, on calcule le volume total des matériaux disparus entre 1985 et 1986 , qui correspond à $41 \mathrm{~mm}$ d'ablation; ce chiffre est à tempérer, étant surtout représentatif du secteur amont des versants.

\subsection{La télémétrie sans réflecteur}

Pour des valeurs données de la direction du faisceau laser calées sur le théodolite, la télémétrie donne directe- ment les distances, à une année d'intervalle. Deux types de traitement ont été utilisés pour comparer ces distances :

- le calcul de la distance entre chaque point $A$ de la surface 1986 et la surface 1985 représentée par le plan des « moindres carrés " passant par les 9 points 1985 les plus proches de $A$. Les mesures ont été effectuées pour les visées de faible incidence sur la facette donnée. Lorsque cet angle dépasse $40^{\circ}$, l'incertitude sur la mesure atteint $1 \mathrm{~cm}$. De plus une imprécision sur le résultat est due au fait que les 9 points utilisés ne sont pas, en général, sur un plan. Elle est réduite par le nombre élevé de mesures qui diminue le bruit à quelques $\mathrm{mm}$. Un biais subsiste, dû à l'utilisation successive d'appareils différents. Cet artéfact, inexistant avec le même matériel, est à l'origine d'une imprécision de 2 ou $3 \mathrm{~mm}$. Les résultats montrent une assez grande variabilité et si des valeurs positives de la distance, indiquant un dépôt, sont au pied des versants, certaines se trouvent dans la partie amont, traduisant une imprécision de la mesure ou un gonflement différent des altérites, lors des deux relevés. En l'absence d'autres mesures, on a effectué la moyenne de toutes les valeurs obtenues. Les résultats indicatifs sont présentés dans le tableau 1. Les ablations verticales totales pour les stations haute et basse sont de $-29,3 \mathrm{~mm}$ et $-17,6 \mathrm{~mm}$;

- pour chaque triplet de droites de visées adjacentes, le calcul des volumes élémentaires (ablation ou dépôt) séparant les surfaces 1986 et 1985 et celui des surfaces des facettes donnent les résultats suivants (tabl. 2).

\section{Discussion}

\subsection{Les contraintes opérationnelles}

La photogrammétrie est rapide sur le terrain, si l'on a pris soin de mettre suffisamment de repères fixes dans le paysage photographié. En revanche, le dépouillement des clichés est fait par des spécialistes et prend du temps (une semaine pour le site test). Par ailleurs, le nombre de points mesurés étant illimité, on améliore la précision de la mesure individuelle à moins de $1 \mathrm{~mm}$. Enfin, un bon éclairage naturel (lumière diffuse) est nécessaire pour éviter les ombres portées. 
Les mesures de télémétrie sans réflecteur sont plus longues et fournissent environ 500 points par jour. Par contre, elles sont directement interprétables par l'usager sans faire appel à un spécialiste. La précision $(5 \mathrm{~mm})$, qui pose des problèmes lorsque l'incidence de la visée sur le sol est rasante, limite un peu l'emploi de la méthode, dont le matériel peut être loué ou acheté. En connectant celui-ci à un micro-ordinateur de terrain, on limite la manipulation des données au minimum. Enfin, pour un suivi des lieux difficiles d'accès ou lors des surveillances fréquentes, cet ensemble peut être automatisé (théodolite motorisé).

Il faut signaler que les deux méthodes sont limitées par la présence de végétation, d'autant plus que les visées se font du sol et non d'hélicoptère.

\subsection{Analyse d'erreurs}

En photogrammétrie, l'erreur atteint quelques cents millièmes de la dimension de l'objet, et les irrégularités liées au grain local sont lissées par l'opérateur. A la Roubine, la précision d'appréciation de la surface du sol est de 1 à $2 \mathrm{~mm}$.

En télémétrie, les dimensions du faisceau interviennent dans la précision des relevés. La mesure représente en fait le barycentre des distances sur tous les points éclairés pondérés par leur albedo. Il y a intérêt à avoir une tache d'analyse réduite, un faisceau proche de l'incidence normale et à employer toujours le même appareil. Pour la Roubine, la précision de mesure est de $5 \mathrm{~mm}$, tant que l'incidence de la visée n'excède pas $30^{\circ}$ et de $1 \mathrm{~cm}$ vers $50^{\circ}$. Notons que l'histogramme des fréquences des angles de visée indique un maximum entre 50 et $55^{\circ}$ ( $f i g .4$ ).

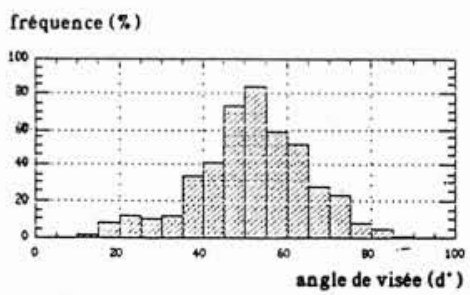

4. Histogramme des fréquences des angles de visée normale.

\subsection{La comparaison des résultats obtenus}

Les résultats des méthodes topographiques ont été comparés à la mesure des transports solides pendant la même période $\left(15,8 \mathrm{~m}^{3}\right)$. Pour cela, on a créé un modèle d'érosion qui établit les relations entre les variables. On a admis une limite tranchée entre la roche saine et les altérites qu'elle génère et supposé que les densités des dépôts, de la roche et des altérations sont constantes, dans l'espace et le temps. Les moyennes temporelles et spatiales sont respectivement surlignées et mises entre crochets. Les notations sont les suivantes $(t=t e m p s$,
$M=$ point d'abscisse $x, y) ; Z_{s}(t, M)=$ altitude du sol au temps $t$ et au point $M ; Z_{R}(t, M)=$ altitude de la roche saine au temps $t$ et au point $M ; P(t, M)=$ épaisseur verticale d'altération $\left(p=Z_{s}-Z_{\mathrm{R}}\right) ; \rho_{a}, \rho_{d}$, $\rho_{R}=$ densité des altérations, des dépôts et de la roche; $e(t, M)=$ vitesse d'abaissement de l'altitude du sol en place par l'érosion; $a(t, M)=$ vitesse d'abaissement de l'altitude de la limite roche-altérites ; $t_{0}, t_{1}=$ instants de mesure et $V\left(t_{0}, t_{1}\right)=$ volume de matériau solide (de densité $\rho_{d}$ ), mesuré entre $t_{0}$ et $t_{1}$. D'où :

$$
Z_{R}\left(t_{1}, M\right)=Z_{R}\left(t_{0}, M\right)-\int_{t_{0}}^{t_{1}} a(t, M) \mathrm{d} t .
$$

L'abaissement de l'altitude de la roche, donné par le second terme de l'équation (1), provoque un accroissement de l'épaisseur d'altération représenté par :

$$
\frac{\rho_{R}}{\rho_{a}} \cdot \int_{t_{0}}^{t_{1}} a(t, M) \mathrm{d} t .
$$

L'épaisseur d'altération et l'altitude du sol au point $M$, au temps $t_{1}$, s'écrivent :

$$
\begin{gathered}
\left(t_{1}, M\right)=p\left(t_{0}, M\right)+\frac{\rho_{2}}{\rho_{a}} \int_{t_{0}}^{t_{1}} a(t, M) \mathrm{d} t-\int_{t_{0}}^{t_{1}} e(t, M) \mathrm{d} t \\
Z_{s}\left(t_{1}, M\right)=Z_{s}\left(t_{0}, M\right)+\left(\frac{\rho_{R}}{\rho_{a}}-1\right) \times \\
\times \int_{t_{0}}^{t_{1}} a(t, M) \mathrm{d} t-\int_{t_{0}}^{t_{1}} e(t, M) \mathrm{d} t .
\end{gathered}
$$

De (3), on déduit $\Delta Z_{s}\left(t_{0}, t_{1}, M\right)$, dont le calcul nécessite de connaître $\langle e\rangle$ mais aussi $\langle a\rangle$. En ce qui concerne $\langle e\rangle$ on a la relation:

$$
\begin{aligned}
V\left(t_{0}, t_{1}\right) & =\frac{\rho_{a}}{\rho_{d}} \int_{t_{0}}^{t_{1}} \int_{M \in S} e(t, M) \mathrm{d} t \mathrm{~d} S \\
& =\frac{\rho_{a}}{\rho_{d}} S \int_{t_{0}}^{t_{1}}\langle e(t)\rangle \mathrm{d} t .
\end{aligned}
$$

A partir de la relation (4) et de la mesure de $V\left(t_{0}, t_{1}\right)$, on obtient une valeur de la moyenne spatiale de l'érosion ; pour la comparer avec les variations d'altitude, il faut utiliser les relations (1), (2), (3) en moyenne spatiale soit : (1bis), (2bis) et (3bis). Avec (4) la relation (3bis) devient :

$$
\begin{aligned}
&\left\langle\Delta Z_{s}\left(t_{0}, t_{1}\right)\right\rangle=\left(\frac{\rho_{R}}{\rho_{a}}-1\right) \int_{t_{0}}^{t_{1}}\langle a(t)\rangle \times \\
& \times \mathrm{d} t-\frac{\rho_{d}}{\rho_{a}} \frac{V\left(t_{0}, t_{1}\right)}{S} .
\end{aligned}
$$

Une estimation de $\langle a(t)\rangle$ est possible avec l'équation (2bis) si l'on mesurait les profondeurs d'altération. Ceci n'ayant pu être fait, on a admis que sur un intervalle de 5 à 10 ans, la fourniture en altérites compenserait leur perte par l'érosion. D'où :

$$
\frac{\rho_{R}}{\rho_{a}}\langle\bar{a}\rangle=\langle\bar{e}\rangle .
$$


Si le rythme moyen $\langle a\rangle$ d'altération de la roche a été suivi entre $t_{0}$ et $t_{1},(5)$ devient :

$$
\begin{aligned}
\left\langle\Delta Z_{s}\left(t_{0}, t_{1}\right)\right\rangle= & \left(\frac{\rho_{R}}{\rho_{a}}-1\right) \times \\
& \times \int_{t_{0}}^{t_{1}}\langle\bar{a}\rangle \mathrm{d} t-\frac{\rho_{d}}{\rho_{a}} \frac{V\left(t_{0}, t_{1}\right)}{S} \\
\left\langle\Delta Z_{s}\left(t_{0}, t_{1}\right)\right\rangle= & \left(\frac{\rho_{R}}{\rho_{a}}-1\right) \frac{\rho_{a}}{\rho_{R}} \times \\
& \times\langle\bar{e}\rangle \cdot\left(t_{1}-t_{0}\right)-\frac{\rho_{d}}{\rho_{a}} \cdot \frac{V\left(t_{0}, t_{1}\right)}{S} .
\end{aligned}
$$

L'équation (7) permet de comparer les mesures topographiques $\left(\left\langle\Delta Z_{s}\left(t_{0}, t_{1}\right)\right\rangle\right)$ aux mesures de transports solides $\left(V\left(t_{0}, t_{1}\right)\right)$, si l'on connaît $\rho_{R}, \rho_{a}, \rho_{d}$ et $\langle e\rangle$.

Pour la période 1984 à 1987 , le volume moyen annuel de l'érosion pour la Roubine est de $11,26 \mathrm{~m}^{3} / \mathrm{an}$. A partir de l'équation (4) et en prenant $\rho_{a}=1,3$ et $\rho_{d}=1,7$, il vient $\langle e\rangle=11,26 \mathrm{~mm} / \mathrm{an}$. Avec $\rho_{R}=2,75, t_{1}-t_{0}=$ 1 an 18 jours $=1,05$ an, $\quad V\left(t_{0}, t_{1}\right)=15,8 \mathrm{~m}^{3}, \quad S=$ $1050 \mathrm{~m}^{2}$, on calcule que $\left\langle\Delta Z_{s}\left(t_{0}, t_{1}\right)\right\rangle=-11,93 \mathrm{~mm}$. Cette valeur est à comparer avec celles obtenues par les méthodes topographiques :

\section{- télémétrie :}

calcul des distances :

stations basse et haute : $-29,3 \mathrm{et}-17,6 \mathrm{~mm}$, calcul des volumes :

stations basse et haute : $-22,7$ et $-13,9 \mathrm{~mm}$.

- photogrammétrie : $-41 \mathrm{~mm}$.

Les écarts entre ces résultats peuvent vraisemblablement s'expliquer par les deux hypothèses qui ont permis de donner des valeurs numériques aux paramètres intervenant dans le modèle théorique développé ci-dessus. Considérées constantes, les valeurs de densité sont hypothétiques, à l'exception de $\rho_{R}$; celles des dépôts $\left(\rho_{d}=1,7\right)$ varie entre 1,5 et 1,8 et celle des altérations ( $\rho_{a}=1,3$ : résultat d'une mesure), doit varier fortement dans le temps. Pour progresser, il faudrait connaître leur évolution et reformuler le modèle pour tenir compte de ces variations. Par ailleurs, la valeur de $a(t, M)$, vitesse d'abaissement de la roche est inconnue; il a fallu admettre qu'entre les 2 dates, elle avait une valeur moyenne déduite des mesures de transport solide sur 4 ans (éq. (6)), ce qui n'est pas certain.

Enfin, il faut rappeler que si les mesures de transport solide traduisent l'érosion sur l'ensemble du bassin, les méthodes topographiques n'en déterminent qu'une partie, ce qui explique, sans doute aussi, les différences entre les résultats de ces deux méthodes, les zones couvertes ne se recouvrant pas exactement.

\section{Bibliographie}

[1] Les Bassins Versants Expérimentaux de Draix. Note de présentation. CEMAGREF, ONF-RTM, $2^{\mathrm{c}}$ version, mai 1988.

[2] Les Bassins Versants Expérimentaux de Draix. Compterendu de recherche $n^{\circ} 1$ en érosion et hydraulique torrentielle. CEMAGREF, ONF-RTM, septembre 1987.

[3] Mathis, M. Menier, C. Guet: Mesure et érosion du processus d'érosion dans les marnes des Alpes du Sud, à l'échelle de la petite ravine. CEMAGREF, La Houille Blanche, n॰ 2, 1989. 